\title{
Polymorphism in a Flagellar-shape Mutant of Salmonella typhimurium
}

\author{
By T. IINO AND TOMOKO OGUCHI \\ Laboratory of Genetics, Faculty of Science, University of Tokyo, Hongo, Tokyo, Japan \\ AND T. KUROIWA \\ Department of Biology, Faculty of Science, Okayama \\ University, Tsushima, Okayama, Japan
}

(Received I4 May I973; revised 20 October I973)

\begin{abstract}
SUMMARY
A flagellar-shape mutant, designated 'polymorphous', was isolated from a normal flagella strain of Salmonella typhimurium. The mutant produces normal flagella in phase I and polymorphous flagella in phase 2. The polymorphous flagella are either straight or possess one of the four distinct wave-forms, namely $\mathrm{M}, \mathrm{S}, \mathrm{N}$ or $\mathrm{C}$, when observed with an electron microscope after negative staining with phosphotungstic acid or uranyl acetate. Conversions between the four wave-forms were found to be brought about mainly by a change in the degree of twisting of longitudinal strands around the axis of a flagella filament, without marked change in the relative lengths of the outermost and innermost strands.

The major fraction of the polymorphous mutant flagella showed the $\mathrm{N}$-form under any conditions of specimen preparation. The remaining four forms appeared as minor fractions in various proportions. Specimens fixed with formalin showed less pronounced polymorphism than unfixed ones. Negative staining with uranyl acetate was more effective than with phosphotungstic acid for observing polymorphism. Even though more than one form appeared among the polymorphous flagella, each individual flagellum comprised a single form except for a rare coexistence of $\mathrm{S}$ and $\mathrm{N}$. The same form of flagella tended to coexist in a bacterium in a heteromorphously flagellated cell population.

It was concluded that the conformation and arrangement of the flagellin molecules responsible for wave-form result from strong mutual interactions between the neighbouring molecules along the flagellar filaments and also, to a lesser extent, between the neighbouring filaments in a flagellar bundle, as well as being influenced by the physico-chemical environment.
\end{abstract}

\section{INTRODUCTION}

A pronounced polymorphism in wave-form has been found among the flagellar filaments reconstituted by copolymerization of straight and normal flagellins of Salmonella when they were mixed in various molar ratios (Asakura \& lino, 1972). A characteristic feature of the polymorphism was that the reconstituted flagellar filaments showed one of five distinct forms depending on the ratio of the two types of flagellins. From these observations and other available data (Asakura, Eguchi \& Iino, I964, I966, I968), it was proposed that the flagellar filaments composed of a single kind of flagellin are themselves polymorphic in nature, with the potentiality to manifest several discrete wave-forms. We report that such a polymorphism is actually observed in a flagellar shape mutant of Salmonella typhimurium. 


\section{METHODS}

Organism. The strain SJ4020 used was isolated as a spontaneous mutant from a diphasic strain of Salmonella typhimurium, SL287. It produces short-waved flagella in phase 2 and normal ones in phase I. Its site of mutation was shown by phage $P_{22}$ transduction to be in the structural gene of phase-2 flagellin, H2. Its motility behaviour in phase 2 is similar to that of the curly flagellar mutants (Iino \& Mitani, I966); the cells rotate, showing a tendency to aggregate in liquid medium, and they cannot spread in semi-solid medium.

Cultivation. The culture media were the same as described by Iino \& Mitani (I966). To obtain phase specific bacterial samples, cultures were initiated in each experiment from a single colony of either spreading type for phase $\mathrm{r}$ or non-spreading type for phase 2, and grown in semi-solid medium. Each colony was transferred to $10 \mathrm{ml}$ of nutrient broth and grown for $\mathrm{I} 8 \mathrm{~h}$ at $37^{\circ} \mathrm{C}$ without shaking. The culture was then divided into two equal parts and $5 \%(\mathrm{v} / \mathrm{v})$ final concentration of formalin was added to one. After $30 \mathrm{~min}$, bacteria were harvested by centrifugation, washed once and finally suspended in deionized water. The final titre of bacteria was adjusted to $10^{8}$ organisms $/ \mathrm{ml}$ and samples were kept for various times up to $3 \mathrm{~h}$ at 7 or $23^{\circ} \mathrm{C}$ until used for electron microscopy.

Electron microscopy. Samples were negatively stained by mixing with equal volumes of either $2 \%(\mathrm{w} / \mathrm{v})$ phosphotungstic acid (PTA) or $2 \%(\mathrm{w} / \mathrm{v})$ uranyl acetate (UA) solution. The final $\mathrm{pH}$ of the mixture was 6.8 or 4.6 , respectively. When a sample remained in a stain solution for a prolonged period, the fraction of flagellar waves with irregular forms increased, especially in UA solution. Therefore 2 to $3 \mathrm{~min}$ after droplets of each sample had been placed onto a thin carbon film mounted on a copper grid, the excess solution was removed by touching the edge of the grid with a piece of filter paper and the sample quickly dried in a vacuum. The grids were examined in a JEM-7A electron microscope. Photomicrographs were taken at a magnification of 5000. As a standard for magnification, polystyrene latex particles (diam 0.7900 $\pm 0.0044 \mu \mathrm{m}$; Dow Chemical Co.) were photographed simultaneously. To check reproducibility, observations were repeated on at least three independent cultures similarly treated.

Statistical analysis of wave-forms. The procedures were basically the same as those of Asakura \& Iino (1972).

RESULTS

Wave-forms of the formalin-fixed specimens

When flagella of phase-2 bacteria of SJ4020 were observed on the specimens fixed with formalin, the contour lengths $(L)$ of their waves were exclusively or predominantly distributed unimodally with the mode at $\mathrm{I} \cdot 0 \mu \mathrm{m}$ (Fig. I, peak N). Wavelengths $(\lambda)$ and heights (h) of the waves were also distributed unimodally (Fig. 2). This group of waves, called the $\mathrm{N}$-form (Fig. 3, N), was commonly observed in formalin-fixed specimens kept in deionized water for various times up to $3 \mathrm{~h}$ at either 7 or $23^{\circ} \mathrm{C}$ and negatively stained with either PTA or UA.

In some specimens, a second (M) form (Fig. 3, M) was detected as a minor fraction with the $L$ mode at $0.5 \mu \mathrm{m}$ (Fig. I, peak M). The modes of $\lambda$ and $h$ in the M-form were also distinctly shorter than those of the N-form (Fig. 2). So far, the M-form has been detected only in specimens kept for $3 \mathrm{~h}$ in deionized water at $23{ }^{\circ} \mathrm{C}$ and negatively stained with UA. The maximum frequency of the M-form in the observed waves was $10 \%$.

In addition to these two forms, up to $5 \%$ of the observed flagella appeared straight (Fig. 3, O). 


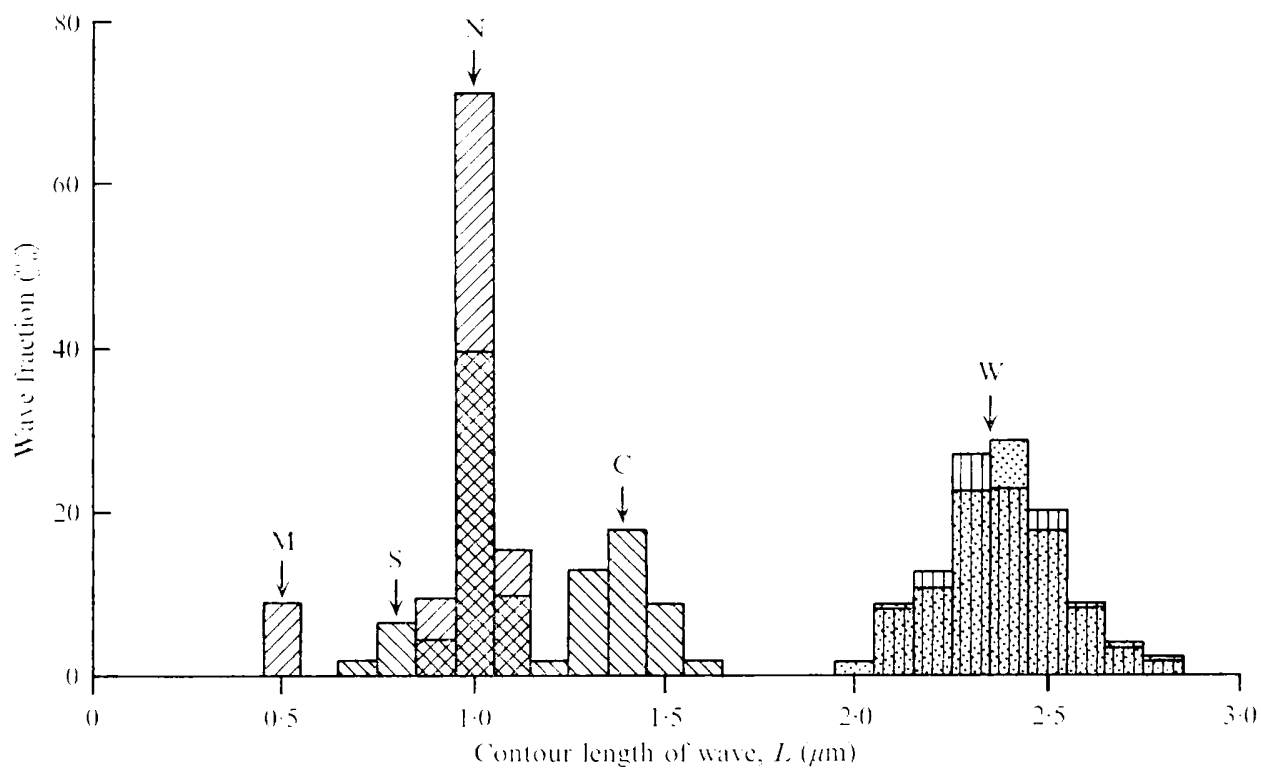

Fig. I. Distribution of contour lengths of waves $(L)$ among flagella in Salmonella typhimurium SJ4020. U and $\bigotimes$, specimens of phase-I and phase-2 bacteria respectively (sample nos.: 106

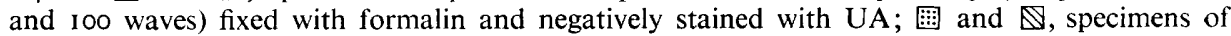
phase-I and phase-2 bacteria respectively (sample nos.: 100 and 2 II waves) kept for $3 \mathrm{~h}$ in deionized water at $7{ }^{\circ} \mathrm{C}$ and negatively stained with UA. Straight flagella which appeared in the former phase-2 specimens were excluded. $\mathrm{M}, \mathrm{S}, \mathrm{N}, \mathrm{C}$ and $\mathrm{W}$ indicate mode values of the five wave-forms. Fractions of $0.05 \mu \mathrm{m}$ and above were counted as $0.1 \mu \mathrm{m}$ and the rest ignored.

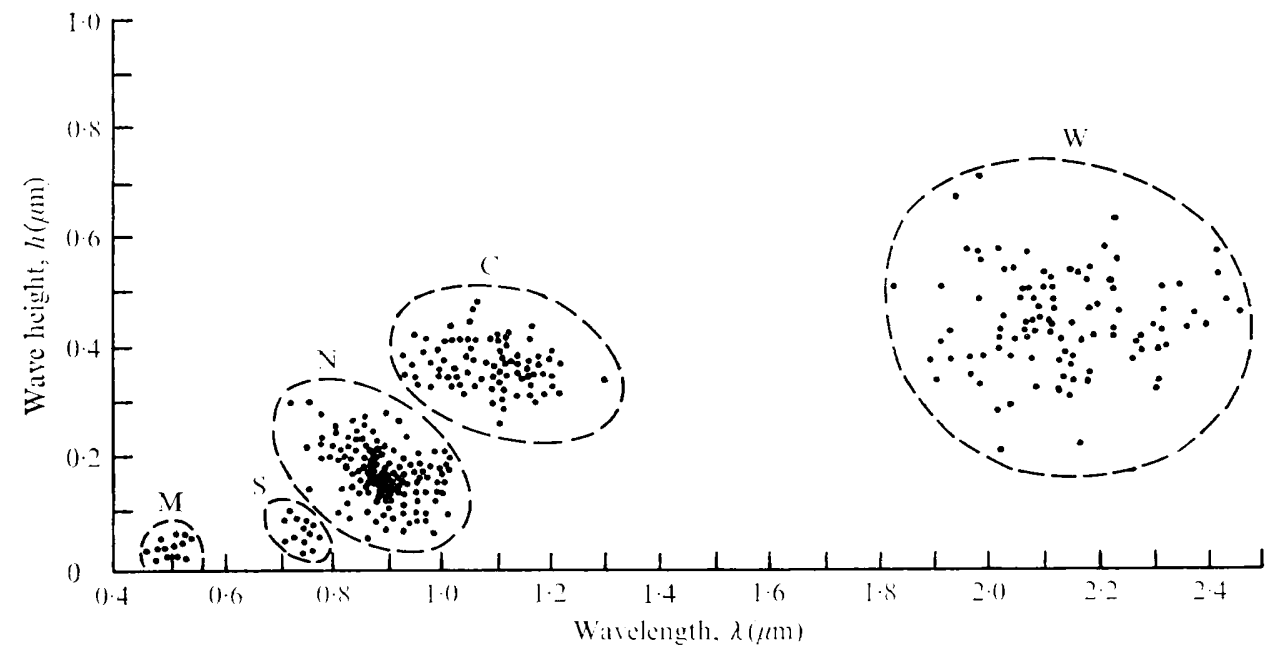

Fig. 2. Distribution of wavelengths $(\lambda)$ and heights $(h)$ of the waves of flagella of the M, S, N, C and $\mathrm{W}$ forms in Salmonella typhimurium sJ4020. Measurements were made on each wave-form from the samples in Fig. I. Each dot denotes a wave. 


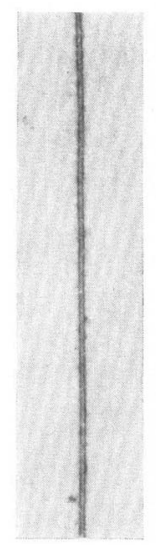

(O)

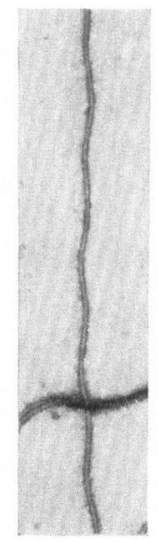

(M)

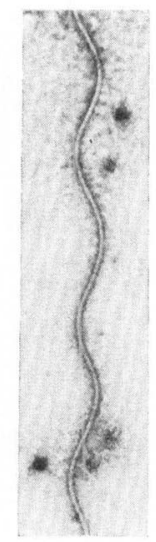

(S)

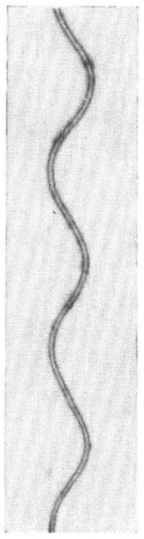

(N)

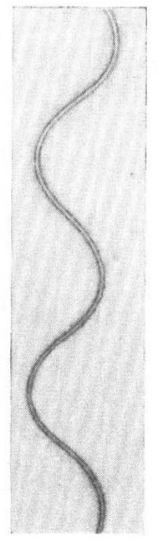

(C)

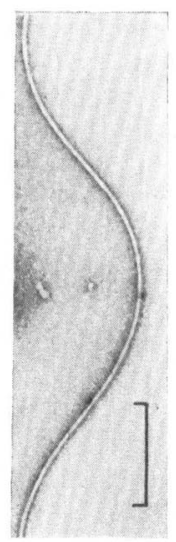

(W)

Fig. 3. Straight form (O) and four wave-forms (M, S, N and C) detected in phase-2 flagella of Salmonella typhimurium sJ4020. W is the normal wave-form in phase I. Bar marker represents $0.5 \mu \mathrm{m}$.

\section{Wave-forms of the specimens prepared without formalin fixation}

Without formalin fixation, more pronounced polymorphism of the flagellar waves was detected than in the formalin-fixed specimens. In unfixed specimens the $\mathrm{N}$-form was predominant. Occasionally, the $\mathrm{M}$-form and the straight-form $(\mathrm{O})$ were detected as minor fractions. In addition to these three forms, two others, each showing unimodal distributions as regards $L, \lambda$ and $h$, appeared in the unfixed specimens (Fig. I, peaks $\mathrm{C}$ and S; Fig. 2 ). The C-form (Fig. 3, C) was characterized by $L, \lambda$ and $h$ being longer than those of the $\mathrm{N}$-form, whereas the S-form (Fig. 3, S) was intermediate between the $\mathrm{N}$ - and $\mathrm{M}$-forms.

The frequency of each form in a sample varied, depending on the method of specimen preparation. When a sample was negatively stained with PTA or UA immediately after suspension in deionized water, more than $90 \%$ of the waves were $\mathrm{N}$-form and the rest were C- and/or S-form. Only rarely did the O-form appear. With an increase in the period of suspension in deionized water, the wave-forms appearing as minor fractions increased proportionally. In specimens kept in deionized water at $23{ }^{\circ} \mathrm{C}$, irregular forms tended to increase for 2 to $3 \mathrm{~h}$ and the distribution of each form became broader. In contrast, specimens kept in deionized water at $7^{\circ} \mathrm{C}$ for up to $3 \mathrm{~h}$ maintained their regular wave-forms without marked deformation. In such specimens, a clear difference in the effects of the stains was noticed: UA enhanced the increase of the C-form fraction, PTA did not. The $\mathrm{M}$-form was detected when the sample was kept for $3 \mathrm{~h}$ in deionized water at $23{ }^{\circ} \mathrm{C}$ and negatively stained with UA, as with the formalin-fixed samples. These differences in the frequency of the five forms among the different specimens were detected even when they were prepared from the same culture.

\section{Effect of low pH on polymorphism}

In the above experiments, negative staining with PTA was carried out at near neutral $\mathrm{pH}(6 \cdot 8)$, while treatment with UA was at low $\mathrm{pH}(4 \cdot 6)$. It was possible that the pronounced polymorphism in phase-2 flagella of $\mathrm{sJ} 4020$ was caused by their exposure to low $\mathrm{pH}$. The phase-2 bacteria were therefore kept for $3 \mathrm{~h}$ at $7{ }^{\circ} \mathrm{C}$ in deionized water without formalin fixation and suspended in $\mathrm{I}^{\cdot} 5 \mathrm{M}$-sodium acetate-acetic acid buffer at $\mathrm{pH} 4 \cdot 6$. After $5 \mathrm{~min}$, 
Table I. Parameters of the four wave-forms exhibited by phase-2 flagella of Salmonella typhimurium $\mathrm{SJ} 4020$

The numbers in parentheses are the standard deviations at the $95 \%$ confidence levels.

$\begin{array}{ccccccc} & L & \lambda & h & \alpha & \Delta \theta \\ \text { Wave-form } & (\mu \mathrm{m}) & (\mu \mathrm{m}) & (\mu \mathrm{m}) & h \lambda & (\%) & \text { (degrees) } \\ \mathrm{M} & 0.52 & 0.50 & 0.04 & 0.08 & 5.53 & 6.76 \\ & (0.02) & (0.02) & (0.01) & & (0.88) & (0.25) \\ \mathrm{S} & 0.76 & 0.72 & 0.10 & 0.14 & 5.05 & 4.48 \\ & (0.02) & (0.02) & (0.01) & & (0.67) & (0.08) \\ \mathrm{N} & 1.01 & 0.92 & 0.16 & 0.17 & 4.77 & 3.30 \\ & (0.02) & (0.02) & (0.01) & & (0.23) & (0.05) \\ \mathrm{C} & 1.38 & 1.10 & 0.36 & 0.33 & 5.52 & 2.09 \\ & (0.02) & (0.02) & (0.01) & & (0.11) & (0.04)\end{array}$

$L=$ contour length of wave; $\lambda=$ wavelength; $h=$ height of wave; $\alpha=$ percentage difference in lengths of the outermost and innermost lines of a flagellar helix; $\Delta \theta=$ average of the angles of the outermost and innermost lines to the axis of a flagellar helix.

droplets of the suspension were placed onto a carbon-coated grid, the excess solution was removed, and the samples were negatively stained with PTA. In the samples thus prepared, more than $86 \%$ of the flagellar waves were $\mathrm{N}, \mathrm{I} 3 \%$ or less were $\mathrm{S}$, and the frequency of either $\mathrm{M}$ or $\mathrm{C}$ did not exceed $\mathrm{I} \%$. These frequencies were identical with those observed for samples negatively stained with PTA without acid treatment. The remarkable increase in $\mathrm{C}$, which was characteristic of the UA-treated samples, did not occur. Therefore the effect of UA on polymorphous manifestation is not attributable solely to exposure to low $\mathrm{pH}$.

\section{Wave-form of the specimens in phase $\mathrm{I}$}

The specimens of phase- I bacteria always possessed normal (W-form) flagella under any preparation conditions applied to samples of phase-2 bacteria. Their contour lengths were distributed unimodally with the mode between 2.3 and $2.4 \mu \mathrm{m}$ (Fig. I, peak W) as were their wavelengths and heights (Fig. 2).

Parameters of the four wave-forms in phase 2

Average values of $L, \lambda, h$ and $h / \lambda$ were calculated on representative specimens of each form except the straight one (Table I). Of the four waves, the M-form resembled the minute form (form IV) which appeared in copolymers of straight-mutant and normal flagellins (Asakura \& Iino, 1972), except that it was more curled. The C-form belonged to the curly group of Leifson (1960). Wavelengths of the $\mathrm{N}$ - and S-forms were also in the range of curly groups of previously described wave-forms. However, when the $h / \lambda$ values of various wave-forms were compared, the overall shape of the $\mathrm{N}$-form was homeomorphic with the normal wave (Fig. 3, W), of which it was the miniature form reduced by a factor of 3. Similarly, the S-form was homeomorphic with the small-amplitude wave reduced by a factor of 3 (Iino \& Mitani, 1966).

The percentage difference in the lengths of the outermost and innermost lines $(\alpha)$ and the average of the angles between the outermost and innermost lines and the axis $(\Delta \theta)$ were calculated on each flagellar helix by the following formulae (Asakura \& Iino, 1972):

$$
\begin{aligned}
x & =\left(2 \pi d / L^{2}\right)\left(L^{2}-\lambda^{2}\right)^{\frac{1}{2}}, \\
\lrcorner \theta & =\pi d \lambda / L^{2},
\end{aligned}
$$


Table 2. Distribution of the wave-forms in phase-2 flagella of Salmonella typhimurium $\mathrm{SJ} 4020$

Specimens of sample I were fixed with formalin and negatively stained with UA; those of sample 2 were kept for $3 \mathrm{~h}$ in deionized water at $7^{\circ} \mathrm{C}$ and negatively stained with UA.

\begin{tabular}{|c|c|c|c|}
\hline \multicolumn{2}{|c|}{ Sample 1} & \multicolumn{2}{|c|}{ Sample 2} \\
\hline $\begin{array}{c}\text { Composition } \\
\text { of wave-form in a } \\
\text { flagellum }\end{array}$ & $\begin{array}{l}\text { No. of } \\
\text { flagella }\end{array}$ & $\begin{array}{c}\text { Composition } \\
\text { of wave-form in a } \\
\text { flagellum }\end{array}$ & $\begin{array}{l}\text { No. of } \\
\text { flagella }\end{array}$ \\
\hline Straight & 2 & - & - \\
\hline $4 \mathrm{M}$ & I & $4 \mathrm{~S}$ & I \\
\hline $5 \mathrm{M}$ & I & ${ }_{5} \mathrm{~S}+\mathrm{I} \mathrm{N}^{*}$ & 2 \\
\hline $2 \mathrm{~N}$ & 24 & $2 \mathrm{~N}$ & 3 \\
\hline $3 \mathrm{~N}$ & 8 & $3 \mathrm{~N}$ & 10 \\
\hline $4 N$ & 7 & $4 N$ & 6 \\
\hline $5 \mathrm{~N}$ & 6 & $5 \mathrm{~N}$ & 4 \\
\hline $6 \mathrm{~N}$ & I & $6 \mathrm{~N}$ & 5 \\
\hline- & - & $7 \mathrm{~N}$ & I \\
\hline - & $\ldots$ & $2 \mathrm{C}$ & I I \\
\hline- & - & ${ }_{3} \mathrm{C}$ & 14 \\
\hline- & - & ${ }_{4} \mathrm{C}$ & 6 \\
\hline$\ldots$ & - & $5 \mathrm{C}$ & 4 \\
\hline
\end{tabular}

* A flagellum composed of five $\mathrm{S}$-form waves and one $\mathrm{N}$-form wave.

Table 3. Combination of the wave-forms in a pair of flagella on a phase-2 cell of Salmonella typhimurium $\mathrm{SJ} 4020$

Specimens were kept $3 \mathrm{~h}$ in deionized water at $7{ }^{\circ} \mathrm{C}$ and negatively stained with UA. On 2I bacteria ( 55 flagella), the wave-forms of flagella were observed on every random combination of pairs of flagella on a bacterium and the frequency of each pair-combination calculated. Theoretical frequencies indicate the expected values if the distributions of flagella with different wave-forms are entirely random among the bacteria.

\begin{tabular}{cccc}
$\begin{array}{c}\text { Combination of } \\
\text { wave-forms }\end{array}$ & \multicolumn{3}{c}{ Frequency of pair-combination $(\%)$} \\
Observed & Theoretical & Difference \\
S-S & 3.8 & 0.4 & +3.4 \\
S-N & 3.8 & $4 \cdot 3$ & -0.5 \\
S-C & 0.0 & 9.4 & -9.4 \\
N-N & 18.9 & $8 \cdot 1$ & +10.8 \\
N-C & 18.9 & $37 \cdot 7$ & -18.8 \\
C-C & 54.7 & 40.1 & +14.6
\end{tabular}

where $d$ is the thickness of the flagellar filament, and average values for $\alpha$ and $\Delta \theta$ were obtained for each wave form (Table I).

The value $0.02 \mu \mathrm{m}$ was adopted for $d$ according to the estimation of O'Brien \& Bennett (1972). Two points emerged from the comparison of these parameters for the four different forms: (i) $\alpha$ does not differ significantly among them; (ii) $\Delta \theta$ increases from 2 to $7^{\circ}$ as the value of $L$ decreases. For O-form flagella, $\alpha=0$ and their $\Delta \theta$ value is unknown.

\section{Distribution of the wave-forms in flagella and bacteria}

Each flagellum of SJ4020 in phase 2 was homogeneously composed of one of the five forms, even though more than one wave-form appeared in a sample. The exceptional heteromorphous flagella were detected in unfixed samples kept in deionized water longer than $2 \mathrm{~h}$ before negative staining (Table 2). They comprised mixtures of the neighbouring forms, $\mathrm{N}$ and $\mathrm{S}$. 
The distribution of flagella with different wave-forms among the bacteria also deviated from random. The observed frequency of coexistence of the same form in a pair of flagella of a bacterium is higher than that expected from a random distribution (Table 3). Among the combinations of two different forms, $\mathrm{S}-\mathrm{N}$ was relatively frequent and $\mathrm{S}-\mathrm{C}$ has not been detected.

\section{DISCUSSION}

Polymorphism of wave-form in a single flagellum has been observed in various bacteria (Leifson, 1960; Iino, 1969). The conversion from one form to another has been known to occur spontaneously (Pijper, I957; Iino \& Mitani, I966) following change of $\mathrm{pH}$ (Leifson, Carhart \& Fulton, I955; Hoeniger, 1965) or formalin fixation (Leifson, 1961). The previously known examples were, however, confined to the manifestation of two alternative forms. Mutant strain SJ 4020 of Salmonella typhimurium is characterized by more extensive polymorphism of flagellar wave in phase 2 : it exhibits five flagellar forms. Because of the extensive polymorphism of flagella, the mutant will be designated 'polymorphous'.

In the sense that manifestation of the flagella polymorphism in SJ402O depends on the method of specimen preparation, it could be regarded as an artifact. Nevertheless, the discreteness and regularity of the five wave-forms indicate that each wave-form represents a stable structure to which the polymorphous flagella can convert. The mutant site of SJ 4020 is in the structural gene for phase-2 flagellin. Therefore, the flexibility of the waveform in SJ 4020 is attributed to the conformational and/or binding instability of its phase-2 flagellin under certain environmental conditions.

A flagellar filament of Salmonella is composed of a number of strands each made up of a chain of flagellin molecules and arranged longitudinally to form a cylinder (Kerridge, Horne \& Glauert, 1962; Lowy \& Hanson, 1965). From studies by electron microscopy, optical diffraction and filtration, the I I longitudinal strands were identified in Salmonella flagella (O`Brien \& Bennett, I972).

To explain the helical form of the flagellar filament, it has been postulated that each of the longitudinal strands has constant helical curvature and they associate side by side so that each strand lies at the same position relative to the outermost or innermost line of the helix at any cross-section of the filament (Lowy \& Spencer, I968; Asakura, 1970). O'Brien \& Bennett (1972) confirmed that the observed angle between the axis of the flagellar filament and its component strands in a straight flagellar mutant of Salmonella typhimurium agreed well with the model of Asakura (1970). According to this model, the percentage difference in length of the outermost and innermost lines $(\alpha)$ represents the difference between the lengths of the outermost and innermost strands, and $\alpha$ is proportional to the curvature of the flagellar helix. It is also said that the average of the angles of the outermost and innermost lines to the axis of the helix $(\Delta \theta)$ represents the degree of twist of the longitudinal strands.

Comparison of the $\alpha$ and $\Delta \theta$ values for different wave-forms of the polymorphous mutant flagella, other than the O-form, revealed some simple geometrical relations, i.e. the relative constancy of $\alpha$ and the increase of $\Delta \theta$ related to the decrease of $L$ in the range 2 to $7^{\circ}$ (Table I). In relation to the argument in the preceding paragraph, these relationships are taken as indications that the four wave-forms in the polymorphous mutant resulted from changes in the degree of twisting of the longitudinal strands around the axis of the filament, without significant changes in the relative lengths of the outermost and innermost strands. As the component longitudinal strands twist more, $L, \lambda$, and $h$ become smaller. A parallel relationship was observed for the polymorphous copolymers of straight-mutant 
and normal flagellins (Asakura \& Iino, 1972). The $\Delta \theta$ values of the polymorphous forms are also in the same range for both the polymorphous mutant flagella and the copolymers. As for the O-form, the straight mutant flagella were identified to be an extreme form, with $L=0$ and $\Delta \theta=7^{\circ}$ (Asakura \& Iino, 1972; O'Brien \& Bennett, 1972). Whether the straight form of the polymorphous mutant flagella is in the same category as that of the straight mutant or is a form of $L=\infty$ is unknown.

The polymorphism in the mutant flagella reported here provides experimental evidence for the previous proposal (Asakura \& Iino, 1972) that homogeneous polymers of a single kind of flagellin themselves are polymorphic in nature, alternating between several discrete stable forms, and that under certain environmental conditions the relative stability of each form is determined by the nature of flagellin. This assumption arose as a consequence of the hypothesis that a flagellin molecule is capable of taking two quasi-stable forms, namely R- and T-type (Asakura, 1970). It was assumed that the latter molecule was slightly longer and more subject to shear deformation than the former, and that the outer strands of a flagellar filament are composed of T-type flagellin and the inner of R-type. Thus, the conversion of wave-form in a flagellar filament resulted from the change of $\mathrm{T}$ to $\mathrm{R}$ or vice versa throughout one or more of the strands. According to this hypothesis, $\alpha$ is regarded as representing the difference in longitudinal lengths of the flagellin molecules of R-type and T-type. Therefore, the relatively constant values of $\alpha$ among the different wave-forms of the polymorphous mutant flagella (Table I) are to be expected, because a single kind of flagellin can acquire definite $\mathrm{R}$ - and T-types with a minor deviation in their lengths within a certain range of the degree of twist of the longitudinal strands.

A remarkable feature of the polymorphous mutant flagella is that a flagellum is exclusively or predominantly composed of a single wave-form even if more than one wave-form appeared in a sample (Table 2). A similar feature has been observed in various dimorphic flagella (Pijper, 1957; Iino \& Mitani, 1967; Asakura \& Iino, 1972). The explanation may be that the neighbouring flagellins along a flagellar filament interact strongly and a part of a filament selects a stable form from those possible, depending on the form of the neighbouring part. In relation to the fact that the same form of flagella tends to coexist in a bacterium in a polymorphous cell population (Table 3), one may recall the bundle formation of flagella in Salmonella (Mitani \& Iino, 1965; lino \& Mitani, I966; Mitani \& Iino, 1968). On a living Salmonella bacterium, peritrichous flagella form a bundle. Interconversion of the helix of a flagellar bundle between two discrete forms was observed in some Salmonella mutants (Mitani \& Iino, 1968), and it was inferred that flagellar filaments in a bundle mechanically interact with each other and so determine their helical forms. This may also apply to the polymorphous flagella in the present report. Thus the conformation and arrangement of flagellin molecules responsible for wave-form are the result of strong mutual interactions between neighbouring molecules along the flagellar filaments and also, to a lesser extent, between neighbouring filaments in a flagellar bundle, as well as being influenced by the physico-chemical environment.

\section{REFERENCES}

Asakura, S. (1970). Polymerization of flagellin and polymorphism of flagella. Advances in Biophysics $\mathbf{~}$, 99-155.

Asakura, S., Eguchi, G. \& Iino, T. (1964). The reconstitution of bacterial flagella in vitro. Journal of Molecular Biology ro, 42-56.

Asakura, S., Eguchi, G. \& IINo, T. (1966). Salmonella flagella: in vitro reconstruction and over-all shapes of flagellar filaments. Journal of Molecular Biology 16, 302-316. 
Asakura, S., Eguchi, G. \& IINo, T. (1968). Unidirectional growth of Salmonella flagella in vitro. Journal of Molecular Biology 35, 227-236.

AsakURA, S. \& IINo, T. (1972). Polymorphism of Salmonella flagella as investigated by means of in vitro copolymerization of flagellins derived from various strains. Journal of Molecular Biology 4, 25I-268.

HoENIGER, J. F. M. (1965). Influence of pH on Proteus flagella. Journal of Bacteriology 9o, 275-277.

IINo, T. (1969). Genetics and chemistry of bacterial flagella. Bacteriological Reviews 33, 454-475.

IIno, T. \& Mitani, M. (1966). Flagella-shape mutants in Salmonella. Journal of General Microbiology 44, 27-40.

IINo, T. \& Mitani, M. (1967). A mutant of Salmonella possessing straight flagella. Journal of General Microbiology 49, 8I-88.

Kerridge, D., Horne, R. W. \& Glauert, A. M. (1962). Structural components of flagella from Salmonella typhimurium. Journal of Molecular Biology 4, 227-238.

Leifson, E. (1960). Atlas of Bacterial Flagellation, p. I7I. New York and London: Academic Press.

LeIFson, E. (I96I). The effect of formaldehyde on the shape of bacterial flagella. Journal of General Microbiology 25, I3I-133.

Leifson, E., Carhart, S. R. \& Fulton, M. (1955). Morphological characteristics of flagella of Proteus and related bacteria. Journal of Bacteriology 69, 73-82.

Lowy, J. \& Hanson, J. (1965). Electron microscope studies of bacterial flagella. Journal of Molecular Biology II, 293-313.

LowY, J. \& SPENCER, M. (I968). Structure and function of bacterial flagella. Symposia of the Society for Experimental Biology 22, 215-236.

Mitani, M. \& IINo, T. (1965). Electron microscopy of bundled flagella of the curly mutant of Salmonella abortivoequina. Journal of Bacteriology 90, 1096-1 10I.

Mitani, M. \& IINo, T. (1968). Electron microscopy of Salmonella flagella in methylcellulose solution. Journal of General Microbiology 50, 459-464.

O'Brien, E. J. \& BenNett, P. M. (1972). Structure of straight flagella from a mutant Salmonella. Journal of Molecular Biology 70, 133-152.

PIJPER, A. (1957). Bacterial flagella and motility. Ergebnisse der Mikrobiologie, Immunitütsforschung und Experimentellen Therapie 30, 37-91. 